\title{
Dense gas formation triggered by spiral density wave in M31
}

\author{
T. Tosaki ${ }^{1}$, Y. Shioya ${ }^{2}$, N. Kuno ${ }^{1}$, K. Nakanishi ${ }^{1}$,

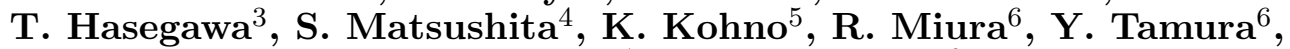 \\ S. K. Okumura ${ }^{1}$ and R. Kawabe ${ }^{6}$ \\ ${ }^{1}$ Nobeyama Radio Observatory, Minamimaki, Minamisaku, Nagano, 384-1805, Japan \\ email: tomoka@nro.nao.ac.jp \\ ${ }^{2}$ Physics Department, Graduate School of Science and Engineering,Ehime University, 2-5 \\ Bunkyo-cho, Matsuyama, Ehime 790-8577 \\ ${ }^{3}$ Gunma Astronomical Observatory, Nakayama, Takayama, Agatsuma, Gunma 377-0702 \\ ${ }^{4}$ Institute of Astronomy and Astrophysics, Academia Sinica, P.O.Box 23-141, Taipei 106, \\ Taiwan, R.O.C. \\ ${ }^{5}$ Institute of Astronomy, University of Tokyo, 2-21-1 Osawa,Mitaka, Tokyo 181-8588 \\ ${ }^{6}$ National Astronomical Observatory of Japan, 2-21-1 Osawa,Mitaka, Tokyo 181-8588
}

\begin{abstract}
We present the high-resolution ${ }^{12} \mathrm{CO}(J=1-0),{ }^{13} \mathrm{CO}(J=1-0)$ and ${ }^{12} \mathrm{CO}(J=3-2)$ maps toward a GMA located on the southern arm region of M31 using Nobeyama $45 \mathrm{~m}$ and ASTE $10 \mathrm{~m}$ telescopes. The GMA consists of two velocity-components, i.e., red and blue. The blue component shows a strong and narrow peak, whereas the red one shows a weak and broad profile. The red component has a lower ${ }^{12} \mathrm{CO}(J=1-0) /{ }^{13} \mathrm{CO}(J=1-0)$ ratio $(\sim 5)$ than that of the blue one $(\sim 16)$, indicating that the red component is denser than the blue one. The red component could be the decelerated gas if we consider the galactic rotational velocity in this region. We suggest that the red component is "post shock" dense gas decelerated due to a spiral density wave. This could be observational evidence of dense molecular gas formation due to galactic shock by spiral density waves.

We also present results from on-going observations toward NGC 604, which is the supergiant HII region of M33, using Nobeyama $45 \mathrm{~m}$ and ASTE $10 \mathrm{~m}$ telescopes. The ratio of ${ }^{12} \mathrm{CO}(J=$ $3-2)$ to ${ }^{12} \mathrm{CO}(J=1-0)$ ranges from 0.3 to 1.2 in $\mathrm{NGC} 604$. The ${ }^{12} \mathrm{CO}(J=1-0)$ map shows the clumpy structure while ${ }^{12} \mathrm{CO}(J=3-2)$ shows a strong peak near to the central star cluster of NGC 604. The high ratio gas is distributed on the arc-like or shell-like structure along with $\mathrm{H} \alpha$ emission and HII region detected by radio continuum. These suggest that the dense gas formation and second generation star formation occur in the surrounding gas compressed by the stellar wind and/or supernova in central star cluster.
\end{abstract}

Keywords. interstellar medium, star formation, spiral arm

\section{Introduction}

It is well established that molecular gas in spiral arms shows very large structures, often referred as Giant Molecular Associations (GMAs; Vogel et al. 1988; Rand \& Kulkarni 1990). Their typical sizes and masses are a few $100 \mathrm{pc}$ and $\sim 10^{7} M_{\odot}$, respectively (Rand \& Kulkarni 1990; Lundgren et al.2004), and observational studies of GMAs in galaxies provide us with invaluable clues on the physics which governs the large scale star formation in the disk regions of galaxies (e.g., Kuno et al. 1995; Tosaki et al. 2003; Sakamoto 1996; Wong \& Blitz 2002; Lundgren et al. 2004). However, GMAs are much larger than Giant Molecular Clouds (GMCs), a major form of molecular contents in our Galaxy, with a typical size and mass of a few $10 \mathrm{pc}$ and $10^{5} M_{\odot}$ (Scoville \& Sanders 1987). Further, 
massive stars are formed in the densest regions of these GMCs, not in the diffuse part of the clouds. Our main interest is to understand the physical process of star formation inside Giant Molecular Associations (GMAs) along the spiral arms, i.e., physical and evolutional links among GMAs (a few 100 pc), GMCs (a few 10 pc), and dense cores ( $\leqslant$ $1 \mathrm{pc}$ ). To address these issues, we carry out the observations of molecular clouds toward the GMA located on the spiral arm of M31 and the supergiant HII region NGC 604 of M33.

\section{Dense gas formation triggered by spiral density wave in M31}

We performed observations of ${ }^{12} \mathrm{CO}(J=1-0)$ and ${ }^{13} \mathrm{CO}(J=1-0)$ emissions toward the south bright arm region of M31 on 2003 December with the 45-m telescope at the Nobeyama Radio Observatory (NRO). The full width at a half-power beam (FWHP) was $16^{\prime \prime}$ and $17^{\prime \prime}$ at rest frequencies of ${ }^{12} \mathrm{CO}(J=1-0)(115.2 \mathrm{GHz})$ and ${ }^{13} \mathrm{CO}(\mathrm{J}=1-0)$ $(110.2 \mathrm{GHz})$, respectively. The size of the observed region is $3^{\prime} \times 4^{\prime}(0.6 \mathrm{kpc} \times 0.8 \mathrm{kpc})$ with a grid spacing of $10 .^{\prime \prime} 3$. We used the 25 BEam Array Receiver System (BEARS), which can simultaneously observe twenty-five positions separated on the sky by $41^{\prime \prime}$ each (Sunada et al. 2000).

We also have carried out observations of ${ }^{12} \mathrm{CO}(J=3-2)$ emission toward a part of the region observed with ${ }^{12} \mathrm{CO}(J=1-0)$ and ${ }^{13} \mathrm{CO}(J=1-0)$ emissions on 2005 August using ASTE 10-m submillimeter telescope located at the Atacama desert in Chile (Ezawa et al. 2004). The beam size at the ${ }^{12} \mathrm{CO}(J=3-2)$ observation was $23^{\prime \prime}$.

The total integrated intensity maps of emission lines are presented in Fig.1. We find that the averaged ${ }^{12} \mathrm{CO}(J=1-0) /{ }^{13} \mathrm{CO}(J=1-0)$ and the ${ }^{12} \mathrm{CO}(J=3-2) /{ }^{12} \mathrm{CO}(J=$ $1-0)$ ratios of the GMA in M31 are 10 and 0.3 , respectively. Large Velocity Gradient calculations show that these ratios correspond to the gas density and temperature of $10^{2-3} \mathrm{~cm}^{-3}$ and $10-20 \mathrm{~K}$, respectively.

The GMA consists of two distinct velocity-components, i.e., red and blue (see Fig.2). The blue component shows a strong and narrow peak, whereas the red one shows a weak and broad profile. The red component has a lower ${ }^{12} \mathrm{CO} /{ }^{13} \mathrm{CO}$ ratio $(\sim 5)$ than that of the blue one $(\sim 16)$. Because LVG calculation also shows that the gas density with the lower ${ }^{12} \mathrm{CO}(J=1-0) /{ }^{13} \mathrm{CO}(J=1-0)$ ratio is larger than that with high ratio at low temperature region such as galactic disk, these ratios indicate that the red component is denser than the blue one. The red component could be the decelerated gas taking into account of the galactic rotational velocity in this region. Based on these results, we suggest that the red component is "post shock" dense gas decelerated due to a spiral density wave. This could be observational evidence of dense molecular gas formation due to galactic shock by spiral density waves.

\section{Dense gas and star formation triggered by first generation star formation in NGC 604}

${ }^{12} \mathrm{CO}(J=3-2)$ observations toward NGC 604 in M33 were performed with ASTE $10-\mathrm{m}$ telescope on July 2006 - August 2006. The size of the observed region is $5^{\prime} \times 5^{\prime}$ $(0.6 \mathrm{kpc} \times 0.8 \mathrm{kpc})$ and we employed On-the-fly method.

We also performed observations of ${ }^{12} \mathrm{CO}(J=1-0)$ toward NGC 604 on 2005 December - 2006 March with the 45-m telescope at NRO. We used the BEARS. The full width at a half-power (FWHP) of beam was $16^{\prime \prime}$ at rest frequency of ${ }^{12} \mathrm{CO}(J=1-0)(115.2 \mathrm{GHz})$. We made a convolution of the map to $25^{\prime \prime}$, to compare with the data by ASTE $10-\mathrm{m}$ telescope. 


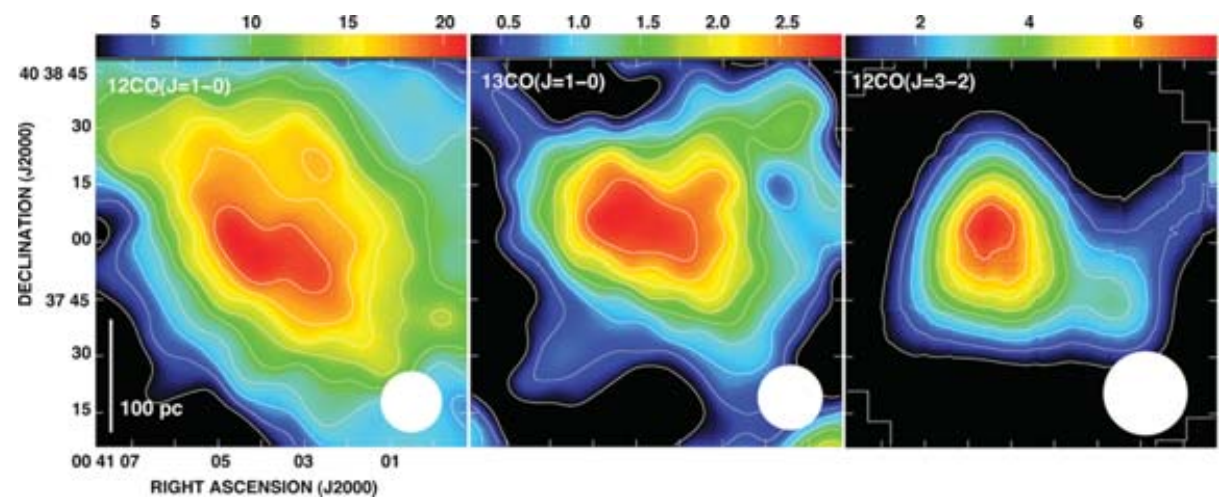

Figure 1. M31 GMA: total integrated intensity maps of ${ }^{12} \mathrm{CO}(J=1-0),{ }^{13} \mathrm{CO}(J=1-0)$ and ${ }^{12} \mathrm{CO}(J=3-2)$. Bottom white filled circles in each panel are beam sizes, corresponding to $54 \mathrm{pc}, 57 \mathrm{pc}$, and $73 \mathrm{pc}$, respectively, at the distance of M31 of $690 \mathrm{kpc}$.

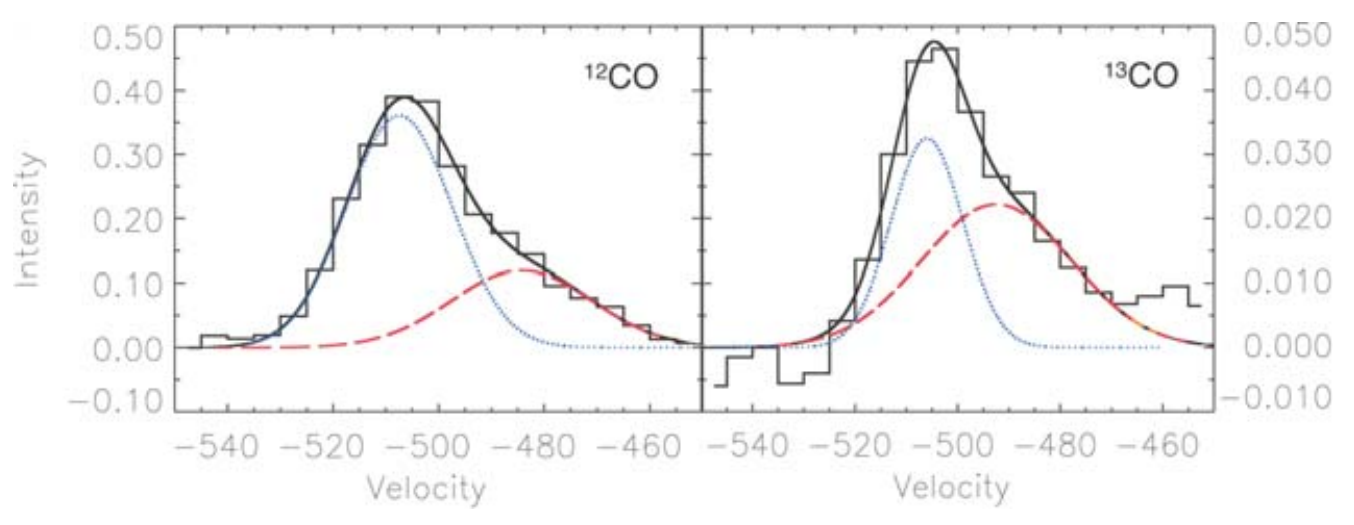

Figure 2. GMA profile of ${ }^{12} \mathrm{CO}(J=1-0)$ and ${ }^{13} \mathrm{CO}(J=1-0)$. Dashed and dotted lines indicate red and blue components. Solid line shows the sum of red and blue components.

The results are shown in Fig. 3. The emission of ${ }^{12} \mathrm{CO}(J=1-0)$ was distributed around NGC 604 and we found several clumpy structures in the map. A typical size of the clumps in the map are $\sim 100$ pc. The molecular clouds around NGC 604 are mainly located at the south of the center of NGC 604, where the central star cluster is located. The southern side corresponds to the upstream side in the NGC 604 with consideration of the galactic rotation of M33.

We found an arc-like or shell like distribution of high ${ }^{12} \mathrm{CO}(J=3-2) /{ }^{12} \mathrm{CO}(J=1-0)$ ratio gas surrounding the central star cluster of NGC 604. The ratio in the arc-like structure is larger than 1. The Large Velocity Gradient calculation suggests that such high ratio is observed in gas with higher temperature than $60 \mathrm{~K}$ and higher density of $10^{3-4} \mathrm{~cm}^{-3}$. In addition, there is also arc-like $\mathrm{H} \alpha$ emission around the central star cluster (Gómez de Castro et al. 2000), where several compact HII regions detected by radio continuum (Churchwell \& Goss 1999) are embedded in them. The shape of the H $\alpha$ distribution shows similarity to that of the high ratio gas distribution. Putting together these facts, the arc-like distribution of warm and dense gas together with on-going star formation is surrounding around central star cluster.

Based on these results, we propose the following scenario; First, stars were formed at the northern part of GMA as "first generation star formation". They are observed as 

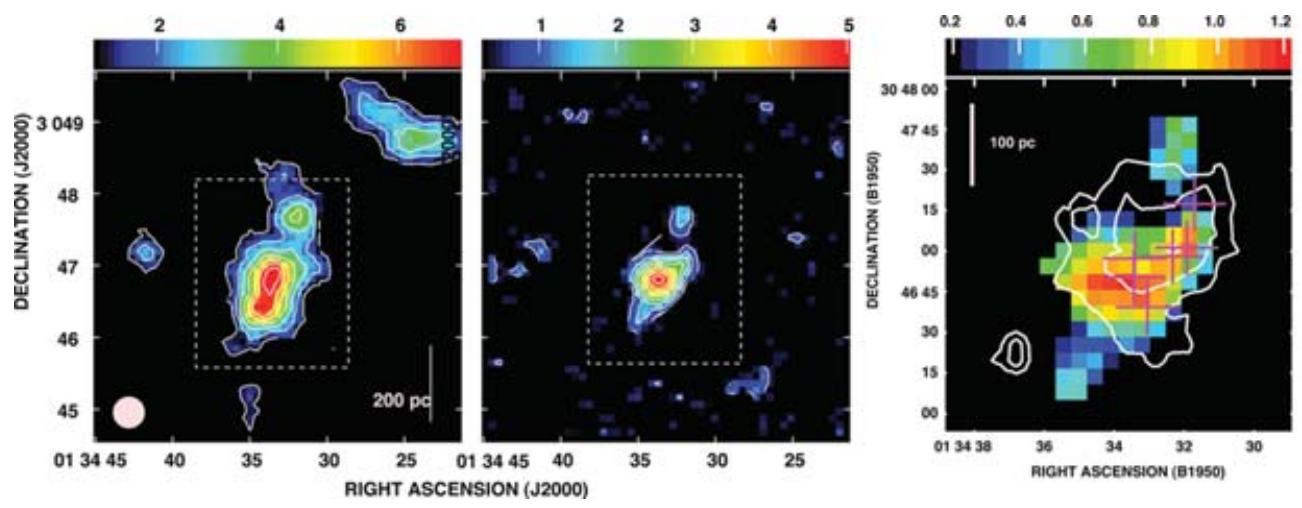

Figure 3. NGC 604: maps of total integrated intensities of ${ }^{12} \mathrm{CO}(J=1-0)$ (left) and ${ }^{12} \mathrm{CO}(J=3-2)$ (middle), and ${ }^{12} \mathrm{CO}(J=3-2) /{ }^{12} \mathrm{CO}(J=1-0)$ ratio (right), respectively. The area of the ratio map is shown as dashed squares in the integrated intensity map. The ${ }^{12} \mathrm{CO}(J=1-0)$ maps were convolved to $25^{\prime \prime}$ beam (white filled circle) which is same as that of ${ }^{12} \mathrm{CO}(J=3-2)$ map. Crosses in the ratio map indicate the HII regions detected by radio continuum.

the central star cluster now. After that, the stellar wind and/or supernova from them compressed surrounding interstellar medium. As a result, dense gas was formed there, and such dense gas is distributed around the central star cluster and shows the high ${ }^{12} \mathrm{CO}(J=3-2) /{ }^{12} \mathrm{CO}(J=1-0)$ ratio. This is observed as arc-like distribution of high ratio gas. And in such dense gas, new stars are forming, and this is "second generation star formation" triggered by first generation star formation. These are seen as a radio compact HII regions now.

\section{Acknowledgements}

We would like to thank the staff of Nobeyama Radio Observatory for their kind support for our observations. The authors wish to express their deep gratitude to Dr. Shigehisa Takakuwa for kindly providing us with his LVG code. This study was financially supported by the MEXT Grant-in-Aid for Scientific Research on Priority Areas No. 15071202. The Nobeyama Radio Observatory is a branch of the National Astronomical Observatory of Japan, the National Institutes of Natural Sciences (NINS).

\section{References}

Churchwell, E. \& Goss, W. M. 1999, ApJ 514, 188

Ezawa, H., Kawabe, R., Kohno, K. \& Yamamoto, S. 2004, SPIE 5489, p. 763

Gómez de Castro, A. I., Sanz, L. \& Beckman, J. 2000, ApESSS 272, 15

Kuno, N., Nakai, N., Handa, T. \& Sofue, Y. 1995, PASJ 47, 745

Lundgren, A. A., Wiklind, T., Olofsson, H. \& Rydbeck, G. 2004, A $\mathscr{J} A$ 413, 505

Rand. R. J. \& Kulkarni, S. R. 1990, ApJ 349, L43

Sakamoto, K. 1996, ApJ 471, 173

Scoville, N. Z. \& Sanders, D. B. 1987, in: D. J. Hollenbach \& H. A. Thronson, Jr. (eds.), Interstellar Processes (Dordrecht: Reidel), p. 21

Sunada, K., Yamaguchi, C., Nakai, N., Sorai, K., Okumura, S. K. \& Ukita, N. 2000, SPIE 4015, 237

Tosaki, T, Shioya, Y., Kuno, N., Nakanishi, K. \& Hasegawa, T. 2003, PASJ 55, 605

Vogel, S. N., Kulkarni, S. R. \& Scoville, N. Z. 1988, Nature 334, 402

Wong, T. \& Blitz, L. 2002, ApJ 569, 157 


\section{Discussion}

BIEGING: Have you considered PDR models to explain the enhanced $\mathrm{CO}(3-2) / \mathrm{CP}(1-0)$ ratios observed in M31 and NGC 604?

TosAKI: Not yet, but we will compare our results with PDR models.

Rosolowsky: When you observe with the millimeter interferometer do you see GMCs preferentially associated with one component of the line seen in the single dish? (Also, you may find the work of Maiz-Apellaniz et al. 2004 (AJ, 128, 1196) relevant to NGC 604 and PDRs). A flux recovery of $<50 \%$ is common for interferometer observations of extragalactic GMCs.

TOSAKI: Yes, we indeed detect two components corresponding to pre-shock and postshock gas in Nobeyama Millimeter Array data. We will also compare our $\mathrm{CO}(3-2) / \mathrm{CO}(1-$ 0 ) results of M33 with PDR models. 